presented a new type of ventricular catheter for its prevention. We also reported the collected cases of ventricular revision by ventricular catheter blockage in Japanese major clinics.

\title{
i-5. Complications in Ventriculo-atrial Shunt and Ventriculo-peritoneal Shunt
}

\author{
Satoru Matsumoto \\ Dept. of Neurosurgery, Kitano Hospital
}

A. J. RaIMONDI

Dept. of Neurosurgery, Cook Country Hospital, Chicago

\section{i-6. Brain Tumors in Children; Surgical Problem and its Solution}

\author{
Yasuhio MatsuKado \\ Department of Neurosurgery Neurological Institute Kyushu University
}

One hundred forty-two cases of central nervous system neoplasms were experienced in patients under 16 years of age during the past 7 years, among which $59 \%$ cases were located in the supratentorial space and $41 \%$ in the infratentorial. Forty cases of brain tumor were found in children under 6 years and also in this age group a slight dominance was noted in the supratentorial space. Brain tumors in infancy were not infrequently masked by hydrocephalus and therefore the congenital hydrocephalus, especially of non-communicating type, should be studied carefully. Because of possible gas embolisation, air study on hydrocephalic patient had been disfavoured if a ventriculo-atrial shunt was indicated as an immediate pressure relieving procedure. However, pneumoencephalography utilizing nitrous oxide solved greatly the disadvantage, since the nitrous oxide is absorbed quickly into the blood stream and exhaled.

Neurosurgical examination for young children required specific cares, and he feasibility of ordinary type of diagnostic procedure was limited unless some nodification was made. Cerebral angiography was safely carried out when ieldinter's catheterisation was utilized. Emulsified oil verntriculography was also nother choice of examination and patients could be kept still in supine position uring the whole procedure. Two dimensional echoencephalography and brain wanning were other procedures facilitating diagnosis of young children, although alese tests were not yet applocable to posterior fossa tumors.

Twelve out of 21 cases of pinealoma received an extensive surgery of andy's approach, and it was indicated that no limitation of surgical accessibility 
existed in regard to young age. Over-all mortality from surgery was $9.2 \%$ and the higher number of death were observed in the cases which missed the chance of surgery, but not in the cases of extensive surgery.

Postoperative metastsis of gliomas in the spinal subarachnoid space or in the ventricles was a threatening complication, and invention of cytostatic agents which might be introduced into the cerebrospinal fluid space without harmful side effects should be urgent necessity.

\title{
i-7. On Complications in Surgery of Hydrocephalus
}

\author{
Hironao Tanabe, Kyo Ishil and Yoshikazu Saito \\ Dept. of Neurosurgery, Tottori University
}

\section{i-8. Four Developmental Defects associated with Cavum Septi Pellucidi: Especially Craniolacunia}

\author{
Tetsuya Leslie Sasabe, Katsuhito Akagi, Yoshikazu Iwata \\ and Masatsugi KobaTaKe \\ Department of Neurological Surgery, Osaka National Hospital \\ Fujio Uemura \\ Department of Pathology, Osaka National Hospital
}

With advent of shunt operation and improvement of antibiotic, prognosis of myelomeningocele accompanied with hydrocephalus has become better. As developmental defects frequently may have several anomalies, the prognosis can not always be expected to be fair. We are reporting such four developmental defects.

(Case 1): A 11-day-old male infant was born with myelomeningocele, hydrocephalus, cavum septi pellucidi, inverted feet, and short toes. At 20 days of age, an operation was performed for the myelomeningocele which showed a total rupture. The infant developed respiratory arrest, expiring 2 hours after the operation. (Case 2): A 2-month-old baby girl came to us with respect to an abnormal swelling at the lumbosacral area. The circumference of the head was within normal limits. Myelomeningocele was operated on first. Four months after the operation the patient's head was noticeably enlarged. "Bubble" pneumoventriculography (PVG) disclosed hydrocephalus, cavum septi pellucidi, and slight Arnold Chiary malformation. Ventriculo-caval shunt was carried out, followed by urinary infection. In addition to abnormalities mentioned above, the patient showed congenital luxation of the right hip joint. At the age of one and 